\title{
Improving PET-Based Physiological Quantification Through Methods of Wavelet Denoising
}

\author{
Jou-Wei Lin, Andrew F. Laine*, and Steven R. Bergmann
}

\begin{abstract}
The goal of this study was to evaluate methods of multidimensional wavelet denoising on restoring the fidelity of biological signals hidden within dynamic positron emission tomography (PET) images. A reduction of noise within pixels, between adjacent regions, and time-serial frames was achieved via redundant multiscale representations.

In analyzing dynamic PET data of healthy volunteers, a multiscale method improved the estimate-to-error ratio of flows fivefold without loss of detail. This technique also maintained accuracy of flow estimates in comparison with the "gold standard," using dynamic PET with O15-water. In addition, in studies of coronary disease patients, flow patterns were preserved and infarcted regions were well differentiated from normal regions.

The results show that a wavelet-based noise-suppression method produced reliable approximations of salient underlying signals and led to an accurate quantification of myocardial perfusion. The described protocol can be generalized to other temporal biomedical imaging modalities including functional magnetic resonance imaging and ultrasound.
\end{abstract}

Index Terms-Multidimensional analysis, myocardial perfusion, positron emission tomography, wavelet denoising.

\section{INTRODUCTION}

I MAGING modalities, such as computed tomography (CT), functional magnetic resonance imaging (fMRI), positron emission tomography (PET), and ultrasonography, have allowed researchers and clinicians to noninvasively evaluate physiological processes within the human body. PET is one of the modalities that acquires sequential images and is a technique for measuring the concentration of positron-emitting tracers within an object [1]. PET is superior to other imaging modalities in determining physiological functions because readily prepared radionuclides, such as oxygen, carbon, and nitrogen, are essential elements of the human body which are well incorporated into normal physiological processes.

With the rapid acquisition of dynamic images, PET not only reveals anatomical information, but can also be used to measure physiological functions in absolute terms. Sequential PET

Manuscript received June 18, 2000; revised August 11, 2000. Asterisk indicates corresponding author.

J.-W. Lin is with the Department of Medicine, National Taiwan University Hospital, Taipei, Taiwan 106. He is also with the Division of Cardiology, College of Physicians and Surgeons, Columbia University, New York, NY 10027 USA.

*A. F. Laine is with the Department of Biomedical Engineering, CEPSR Building, Room 416, 530 West 120th Street, MC 8904, Columbia University, New York, NY 10027 USA (e-mail: laine@ columbia.edu).

S. R. Bergmann is with the Division of Cardiology, Department of Medicine and Department of Radiology, College of Physicians and Surgeons, Columbia University, New York, NY 10027 USA.

Publisher Item Identifier S 0018-9294(01)00137-9. images provide time-activity data of an input function (blood supply of tracers into a target organ) and an output function (tissue activity). The basis of physiological quantification is developing an appropriate model, i.e., a system of linear equations that describes biological phenomena and functions. Physiological quantification with a kinetic model usually divides a target system into several subspaces. Each of these subspaces, or "compartments," is assumed to be homogeneous and well mixed. These compartments interact by exchanging materials. Blood and tissue time-activity data can be fitted to a kinetic model to measure physiological function [2]. The fitting process is usually an iterating optimization and is guided by a least square or maximal likelihood method to find the estimates of unknown parameters.

Due to the need to evaluate myocardial function in a large population of heart disease, PET has evolved as a noninvasive approach for assessing myocardial perfusion and metabolism. For example, extractable (e.g., ${ }^{82} \mathrm{Rb}$ and nitrogen-13 $\left({ }^{13} \mathrm{~N}\right)$ ammonia) [3]-[7] and diffusible (e.g., oxygen-15-labeled $\left({ }^{15} \mathrm{O}\right)$ water) tracers [8], [9] have been used in dynamic PET scans. Myocardial perfusion can be quantified with the application of an appropriate kinetic model.

However, methods of PET-based physiological quantification have not been always successful. For example, rubidium- 82 $\left({ }^{82} \mathrm{Rb}\right)$ is a generator-produced flow tracer with a short physical half-life (76 s) [10]. Sequential scanning can be performed within a short period of time in a place where a cyclotron is not available [11]. Although these characteristics shorten the time span for repeated studies and increase the flexibility in designing a clinical study [12], the short physical half-life results in noisier images. Therefore, dynamic curves derived from ${ }^{82} \mathrm{Rb}$ images are error-prone and consequent flow estimates are usually biased.

Wavelet methods have been previously used to enhance specific features and reduce noise in medical images [13], [14]. However, the effect of such post-processing techniques on PET-based physiological quantification has not been evaluated. The wavelet transform is an analysis tool that expands signals onto orthogonal and semi-orthogonal bases. Since wavelets have their energy well localized in time or space, the wavelet transform is especially suitable for the analysis of nonstationary signals, such as medical image and time-activity data. The energy of a signal is partitioned through a wavelet expansion to express simultaneous time (space) and frequency local information. Compared with conventional methods previously used to smooth PET images [15] or to multidimensional curves, wavelet-based noise reduction offers a unique theoretical advantage for identifying a signal from noise. Wavelet transforms 


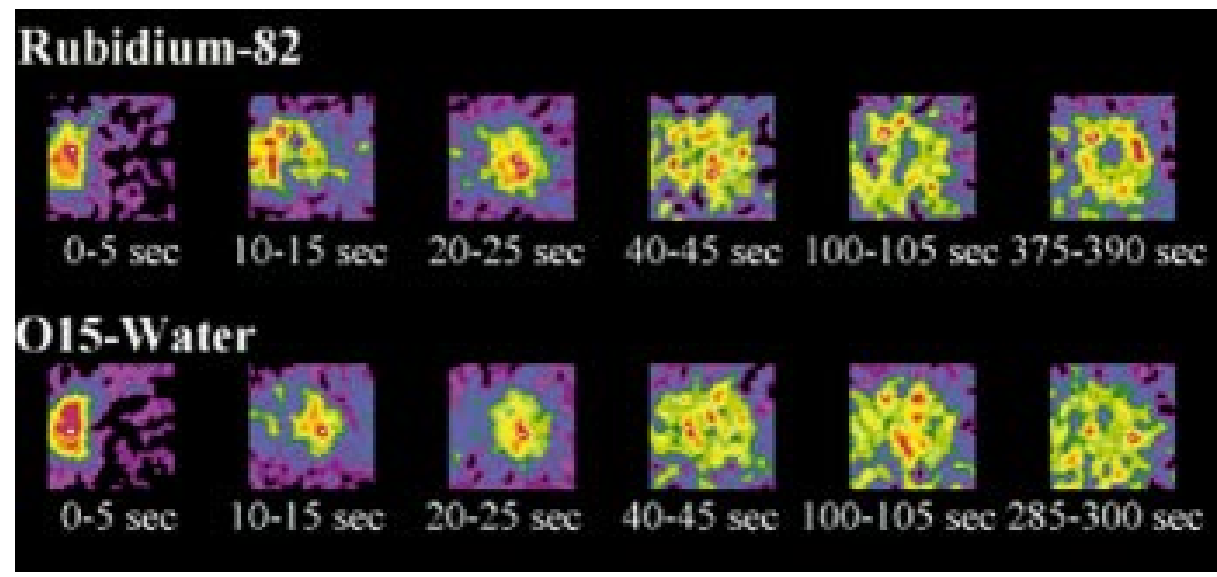

Fig. 1. Sequential images (frames) of a mid-ventricular short-axis plane derived from ${ }^{82} \mathrm{Rb}$ (upper row) and $\mathrm{H}_{2}^{15} \mathrm{O}$ scans (lower row). ${ }^{82} \mathrm{Rb}$ is an extractable tracer. The tracer entered the right ventricle ( $0-5 \mathrm{~s})$, circulated into left ventricle (10-15 s, 20-25 s), then was gradually extracted into the myocardium (20-390 s). $\mathrm{H}_{2}^{15} \mathrm{O}$ is a diffusible tracer. The tracer finally reaches an equilibrium between blood and tissue.

have a built-in capacity of adapting to local changes [16]. With an appropriate soft thresholding algorithm, an estimate is at least as smooth as the true underlying function [17], [26], [27]. Most importantly, there exists a validated method to find a near-optimal risk assessment [18].

The goal of this study was to evaluate specific protocols of wavelet-based noise-suppression methods on improving the signal-to-noise ratio (SNR) of dynamic radioactivity in ${ }^{82} \mathrm{Rb}$ PET images and to demonstrate their robustness for obtaining precise and accurate quantitative results of myocardial perfusion.

\section{METHODS}

\section{A. Subjects}

Eleven volunteers (five males and six females) with a low probability of coronary disease were recruited. Their average age was forty-four. All of the volunteers were placed in a Siemens ECAT-47 PET scanner and initially received a transmission scan with germanium-68/gallium-68 $\left({ }^{68} \mathrm{Ge} /{ }^{68} \mathrm{Ga}\right)$ for attenuation adjustments. A dose of ${ }^{82} \mathrm{Rb}$ (maximum: $0.7 \mathrm{mCi} / \mathrm{kg}$ ) was infused intravenously. A seven-minute scan was performed (36 5-s frames + eight 15-s frames + four 30-s frames). Then, a bolus of $\mathrm{H}_{2}^{15} \mathrm{O}$ (maximum: $0.4 \mathrm{mCi} / \mathrm{kg}$ ) was injected and a five-minute scan was performed (24 5-s frames + six 10-s frames + eight 15-s frames) (IRB\# 7839, Bergmann). These doses were meticulously selected so that the dead time was less than $30 \%$. Seven of them received the same scan again several months later. The total number of studies performed was eighteen.

In addition to healthy volunteers, 13 patients (nine males and four females, mean age: 63) participated in this study. All of them had a history of coronary disease or myocardial infarction and were referred to Columbia Presbyterian Medical Center for evaluation of myocardial viability. They only received the ${ }^{82} \mathrm{Rb}$ scan at rest. The ${ }^{82} \mathrm{Rb}$ protocol was the same as that of the healthy volunteers.

\section{B. PET-Based Quantification of Myocardial Perfusion}

1) Image Analysis: Emission PET data were reconstructed to form cardiac images and then reoriented into short-axis images. A heart was typically divided into 6-10 horizontal planes according to its size. Therefore, a dynamic PET scan would produce four-dimensional images, i.e., a stack of horizontal planes over time. A sample of sequential images of a mid-ventricular plane derived from ${ }^{82} \mathrm{Rb}$ and $\mathrm{H}_{2}^{15} \mathrm{O}$ scans are shown in Fig. 1.

2) Region of Interest (ROI) Operation: The cardiac area (myocardium) of each PET image was grouped into ROIs. The last frame of each ventricular plane was used to determine the position of each region. The outer and inner contours of the heart were separated by $1.0 \mathrm{~cm}$ (five pixels) and centered about the peak of myocardial circumferential activity [7]. Each mid-ventricular slice was equally divided into eight sectors, each of which was of a fan shape (Fig. 2). The pixel intensity within each ROI was then averaged.

A two-pixel-by-two-pixel square near the center of the left ventricular chamber was also selected (Fig. 2). The mean intensity of these four pixels indicated the amount of blood radioactivity at the given frame.

3) Blood and Tissue Time-Activity Curves: Since blood and tissue regions of interest were selected from the last frame, the same template was applied to previous frames of the mid-ventricular plane. The radioactive intensity of pixels within each region at any given frame was then averaged. Since there were sequential frames, the average intensity of the same region over time constituted a time-activity curve. One or two blood curves were identified for each plane. A single blood time-activity curve that averaged all of the individual blood curves was used as the input function in a kinetic model described below. Fig. 3 shows the blood and tissue time-activity curves of a selected ROI.

The collection of tissue time-activity curves of all ROIs was defined as an "observed activity matrix." For example, the observed activity matrix was an $8 \times N \times 48$ matrix for a typical ${ }^{82} \mathrm{Rb}$ study (8 ROIs/plane, $N=6$ to 10 planes/heart, 48 frames) and an $8 \times N \times 38$ matrix for a $\mathrm{H}_{2}^{15} \mathrm{O}$ study (38 frames). Each 


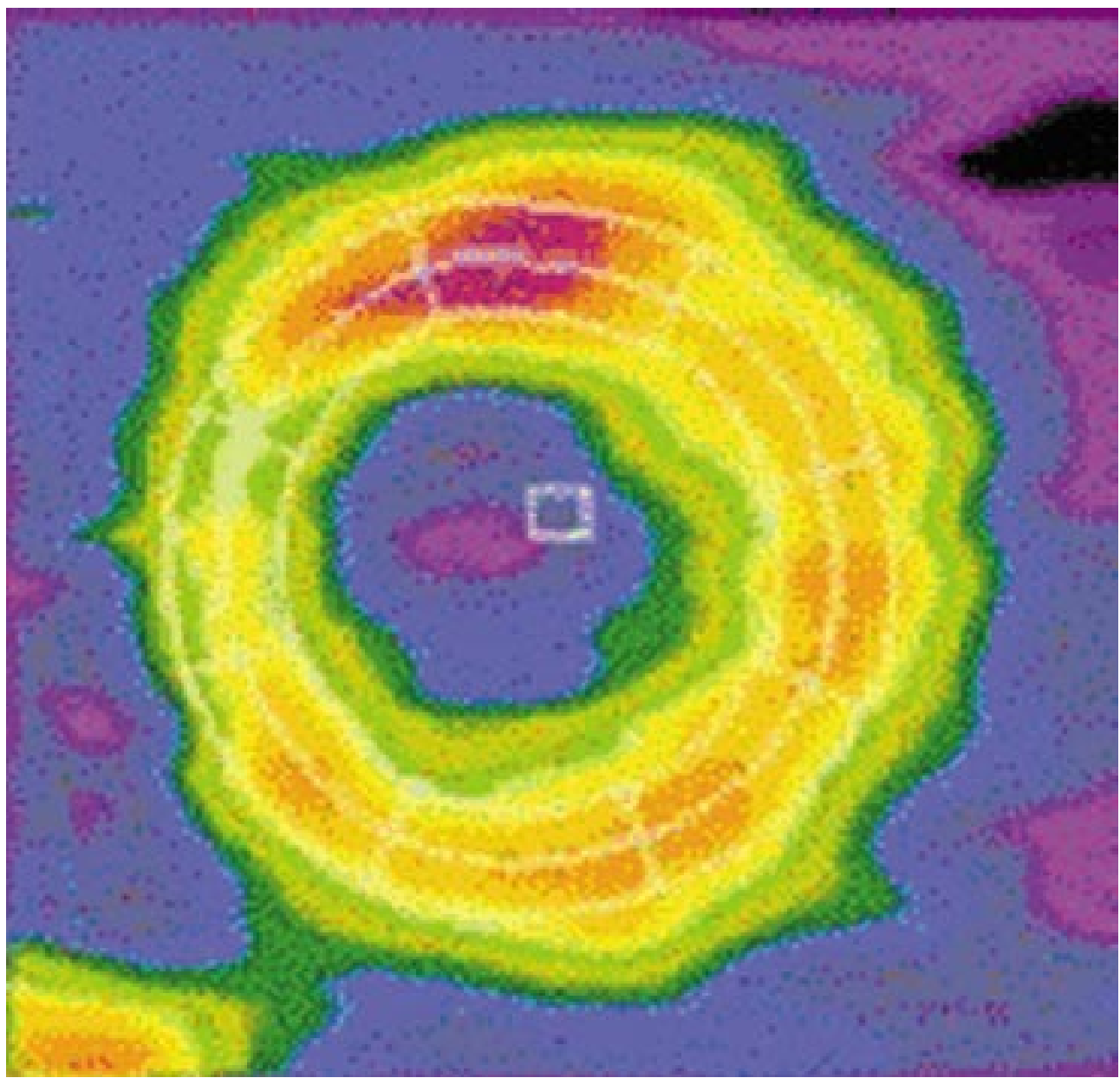

Fig. 2. Last frame of a mid-ventricular plane that shows a short-axis slice of the heart. A circumferential analysis determined the center of the peak activity within the myocardium (middle white circle), the outer (outer white circle) and inner contour (inner white circle) of the heart. The myocardium in this plane was equally divided into eight regions of interest. A square near the center of the left ventricular chamber was selected to show blood activity.

\section{Dynamic Curves}
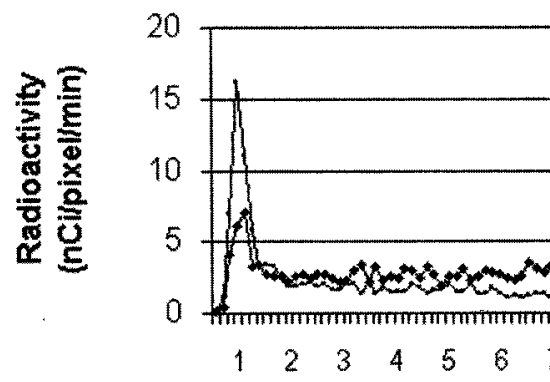

Time (minutes)

Fig. 3. Blood (thin line) and tissue (thick line) time-activity curves of a ROI derived from a mid-ventricular plane of a ${ }^{82} \mathrm{Rb}$ scan.

element contained the mean intensity within a ROI at a given time. Each $8 \times N$ submatrix recorded the global radioactivity of the heart in a certain frame, while each 48-element array represented a tissue time-activity curve (Fig. 4). The observed activity matrix encapsulated the spatial relationships among ROIs and the temporal relationships among sequential frames.

4) Model Fitting: To measure myocardial flow, a two-compartment model previously developed [19] to describe the ki-

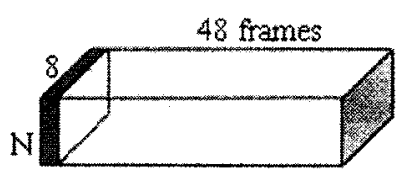

(a)

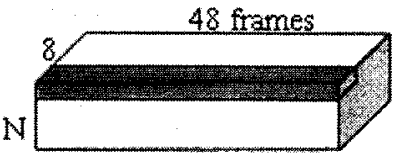

(b)
Fig. 4. An observed activity matrix was derived from a ROI operation on dynamic images. Typically, an $8 \times N \times 48$ matrix for ${ }^{82} \mathrm{Rb}$ studies and an $8 \times N \times 38$ matrix for $\mathrm{H}_{2}^{15} \mathrm{O}$ studies were constructed. (a) Each $8 \times N$ submatrix recorded the global activity of the entire heart. (b) Each 48-element array represented a tissue time-activity curve for a specific ROI.

netics of ${ }^{82} \mathrm{Rb}$ was used. As shown in Fig. 5, the two compartments are the extracellular space (intravascular and interstitial space) and the intracellular space. The system is modeled by the following set of differential equations:

$$
\begin{aligned}
d Q_{1}(t) / d t= & -\left(K_{1}+\mathrm{MBF}\right) / V * Q_{1}(t) \\
& +K_{2} * Q_{2}(t)+\mathrm{MBF} * C a(t) \\
d Q_{2}(t) / d t= & K_{1} / V * Q_{1}(t)-K_{2} * Q_{2}(t) \\
Q(t)= & Q_{1}(t)+Q_{2}(t)+\mathrm{SP} * C a(t) .
\end{aligned}
$$

The input function $\mathbf{C a}(t)$ was the time-activity curve of the left ventricle. The output function $\mathbf{Q}(t)$ reported observed regional activities derived from each dynamic image sequence. 


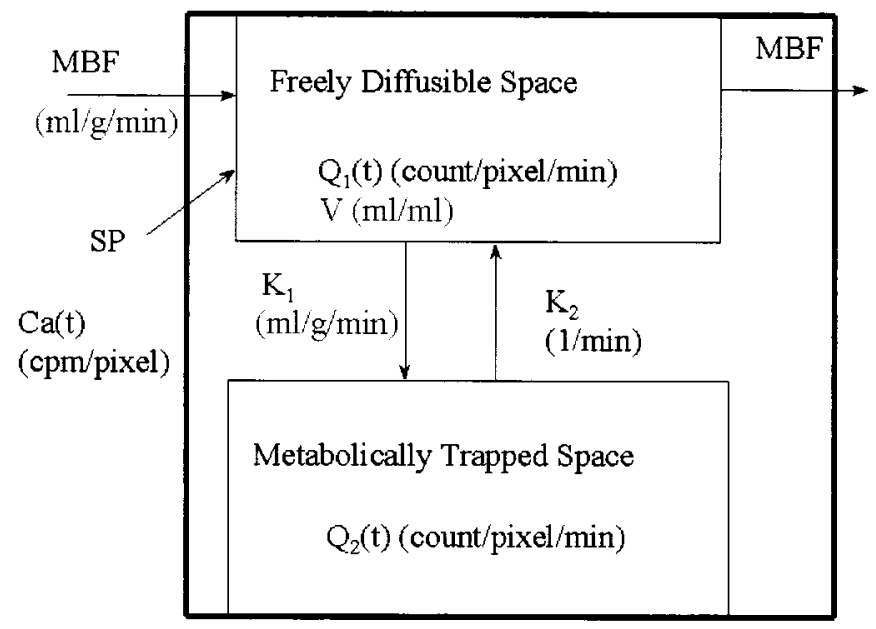

Fig. 5. Two-compartment model of ${ }^{82} \mathrm{Rb}$.

Four parameters were estimated: myocardial blood flow (MBF in $\mathrm{ml} / \mathrm{g} / \mathrm{min}$ ), spillover (SP), forward rate constant ( $\mathrm{K} 1$ in $\mathrm{ml} / \mathrm{min} / \mathrm{g}$ ), and backward rate constant (K2 in $1 / \mathrm{min}$ ). The recovery coefficient $(\mathrm{RC})$ was fixed at 0.65 , a value that was found in previous ${ }^{13} \mathrm{~N}$-ammonia studies. The fraction of volume distribution $(V)$ was set to $0.75 \mathrm{ml} / \mathrm{ml}[6]$.

To measure flow with the diffusible tracer $\mathrm{H}_{2}^{15} \mathrm{O}$, a previously developed one-compartment model was used (Fig. 6). The turnover rate of the tracer's concentration is proportional to both myocardial perfusion and the radioactivity gradient over the arterial and venous sides of capillaries [(4) and (5)] [8]

$$
\begin{aligned}
d Q_{1}(t) / d t & =\mathrm{MBF} / \mathrm{V}(C a(t)-C v(t)) \\
Q(t) & =Q_{1}(t)+\mathrm{SP} * C a(t)
\end{aligned}
$$

The input blood curve $\mathbf{C a}(t)$ and tissue curve $\mathbf{Q}(t)$ were derived from the ROI operation on PET images with $\mathrm{H}_{2}^{15} \mathrm{O}$. The tissue/blood partition coefficient was set to 0.92 [9]. Three parameters were estimated by the one-compartment model: myocardial blood flow (MBF, $\mathrm{ml} / \mathrm{g} / \mathrm{min})$, recovery coefficient (RC), and spillover (SP).

Parameters in the above two models were quantified by minimizing the weighted least-square error between the observed curve and the expected curve. The error was weighted by the inverse of the tissue time-activity curve [20]. Euler's method was used to integrate expected tissue curves according to each model. The optimal estimates were found by an iteration process, which was guided by Marquardt's algorithm [21]. The iteration process did not stop until convergence criteria were met or the number of iterations exceeded 300.

Variation in the point estimates of the parameters was computed as the asymptotic standard errors which were derived from the diagonal elements of the variance-covariance matrix as shown below:

$$
\mathrm{SE}_{i}=\operatorname{SQRT}\left[Q /(n x-n p) \times B^{T} W B_{i j}^{-1}\right]
$$

where

$\mathrm{SE}_{i} \quad$ standard error of parameter $i$;

$n x \quad$ number of data points;

$n p \quad$ number of parameters;

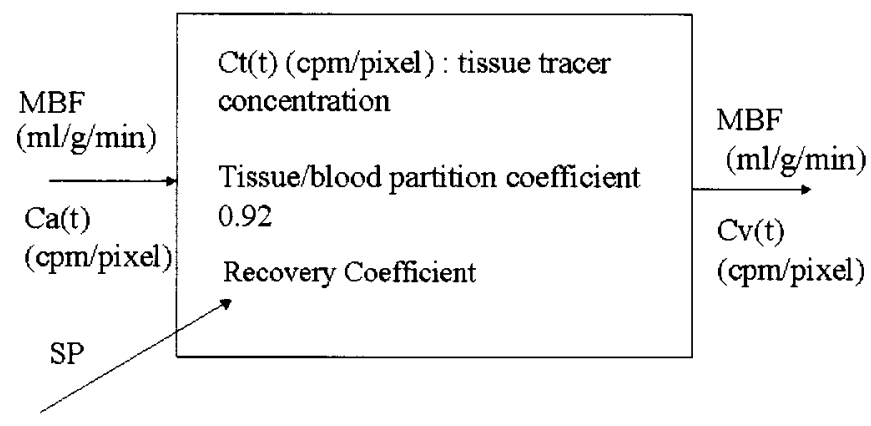

Fig. 6. One-compartment model of $\mathrm{H}_{2}^{15} \mathrm{O}$, used to estimate myocardial perfusion.

$Q \quad$ sum over all $n x$ observations of the squared difference between the model predictions and observed data:

$B^{T} W B n p \times n p$ matrix whose elements $B^{T} W B_{i j}$ are the sum over all $n x$ observations of the weights times the derivative of the model response with respect to parameter $i$ times the derivative of the model response with respect to parameter $j$.

Only the diagonal elements of the inverse matrix are used for computing the standard error of the estimates.

5) Presentation of Counts and Functional Data: Both the PET scans and the quantitative results can be presented by a polar map (or bull's eye). A polar map is a two-dimensional (2-D) representation of the global activity and consists of concentric circles. The inner circles show activity near the cardiac apex, and the outer circles show activity near the base. The right, lower, left, and upper parts of a polar map correspond to the lateral, posterior, septal, and anterior walls of the heart. A count-based polar map represents the cumulative radioactivity (e.g., third through seventh minute in ${ }^{82} \mathrm{Rb}$ scans) of the heart (Fig. 7).

In addition to radioactivity counts, a polar map can be used to represent absolute flows derived from model fitting. A "functional polar map" can provide quantitative information about the amplitude and distribution pattern of flows in the heart from the first glimpse. Fig. 8 shows an example of a functional polar map derived from a patient's ${ }^{82} \mathrm{Rb}$ scan.

\section{Wavelet Denoising Protocols}

1) Wavelet Representations: The multiscale expansion used in this study was generated from scaling and wavelet functions [22], [23] by dyadic dilation and translation. The Fourier transforms of the scaling and wavelet functions used in this study are shown in (7) and (8), respectively. The scaling factor $j$ is effective for determining the frequency and the translation factor $k$ determines the localization in time or space [(9) and (10)]

$$
\begin{aligned}
\hat{\Phi}^{(\omega)} & =(\sin (\omega / 2) /(\omega / 2))^{3} \\
\hat{\Psi}^{(\omega)} & =(i \omega)^{r}(\sin (\omega / 4) /(\omega / 4))^{4} \\
\Phi_{j, k}(t) & =2^{j / 2} \Phi\left(2^{j} t-k\right) \\
\Psi_{j, k}(t) & =2^{j / 2} \Psi\left(2^{j} t-k\right) .
\end{aligned}
$$

A signal $\mathbf{f}(\mathbf{t})$ is decomposed into a weighted sum of wavelet and scaling coefficients at a preselected number of levels of 


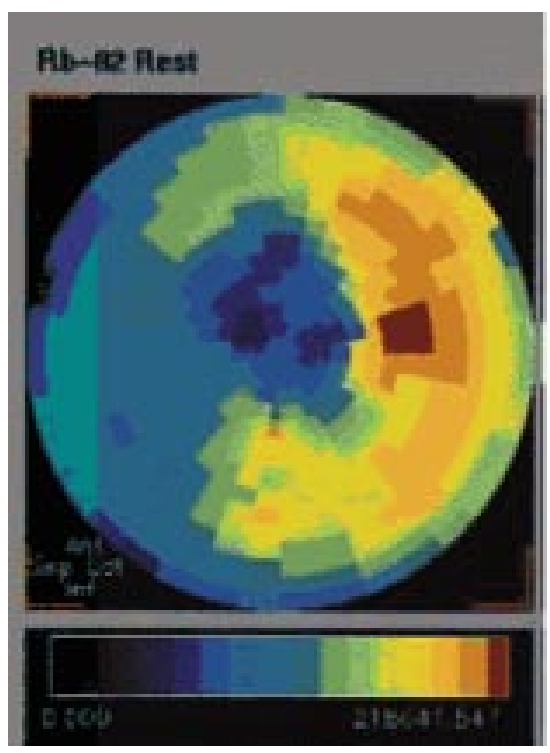

(a)

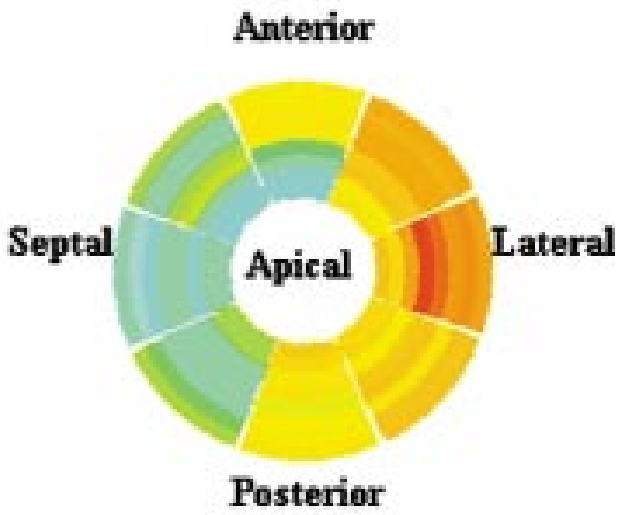

(b)

Fig. 7. (a) Count-based polar map of a ${ }^{82} \mathrm{Rb}$ scan produced by a Siemens ECAT system and (b) regrouped after the ROI operation. The color scale represents cumulative radioactivity in each region.
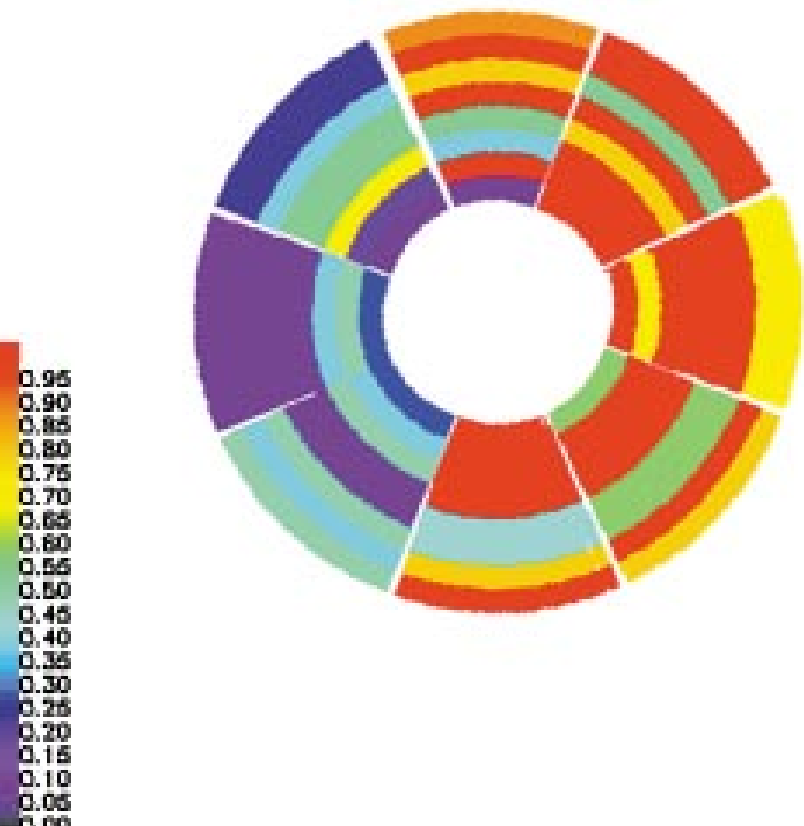

Fig. 8. Functional polar map of a ${ }^{82} \mathrm{Rb}$ scan that shows absolute flow information derived from the two-compartment model. Colors are scaled from 0.0 to $1.0 \mathrm{ml} / \mathrm{g} / \mathrm{min}$.

analysis and positions. In dealing with finite (windowed and sampled) biological signals, an expansion of $\mathbf{f}(\mathbf{t})$ can be efficiently computed by a discrete wavelet transform [(11)]

$$
f(t)=\sum_{k} c_{j 0}(k) \Phi_{j 0, k}(t)+\sum_{k} \sum_{j 0}^{j 1} d_{j}(k) \Psi_{j, k}(t) .
$$

The real numbers $c_{j 0}$ are scaling coefficients at distinct locations within the lowest scale $j_{0}$. The array $d_{j}(k)$ contains wavelet coefficients describing space-frequency information at scale $j$. The depth of multiscale analysis ranged from $j_{0}$ to $j_{1}$ according to the number of samples.
The wavelet expansion was implemented as an "iterated filter bank." Scaling coefficients and wavelet coefficients at lower scales were derived from the scaling coefficients at higher scales of analysis by a convolution with Finite Impulse Response (FIR) filters. Equations (12), (13), (14), and (15) list the discrete coefficients of the FIRs of the scaling and wavelet functions, respectively used in this study. The impulse response $\mathbf{h}(\mathbf{n})$ was a low-pass filter that generated the scaling coefficients. The impulse response $\mathbf{g}(\mathbf{n})$ was a high-pass filter that produced wavelet coefficients of lower scales from coefficients of higher scales. The impulse response $\mathbf{k}(\mathbf{n})$ reversed the process of $\mathbf{g}(\mathbf{n})$ during synthesis. The filter $\mathbf{h}^{*}(\mathbf{n})$ denotes the complex conjugate of $\mathbf{h}(\mathbf{n})$ and was an FIR during synthesis. Due to the symmetry of the low-pass filter, $\mathbf{h}(-\mathbf{n})$ was used as $\mathbf{h}^{*}(\mathbf{n})$ in a one-dimensional (1-D) operator for wavelet synthesis (reconstruction). In dealing with a 2-D image, an additional finite impulse response filter $\mathbf{l}(\mathbf{n})$ was applied during synthesis to each lower frequency channel in order to achieve amplitude normalization

$$
\begin{aligned}
& h(-2,-1,0,1)=[0.125,0.375,0.375,0.125] \\
& g(-1,0)=[2,-2] \\
& k(-2,-1,0,1,2,3) \\
& \quad=[-0.0078125,-0.0546875,-0.1718175, \\
& \quad 0.1718175,0.0546875,0.0078125] \\
& l(-3,-2,-1,0,1,2,3) \\
& =[0.0078125,0.046875,0.1171875,0.65625, \\
& \quad 0.1171875,0.046875,0.0078125] .
\end{aligned}
$$

The wavelet representations used in this study were intentionally constructed to be redundant [24]. Thus, down-sampling and up-sampling were not performed during wavelet expansion and synthesis, respectively. The over-complete representation ensured shift-invariance and avoided the possible introduction of aliasing artifacts [25]. A schematic of the general algorithm 


\section{Expansion}

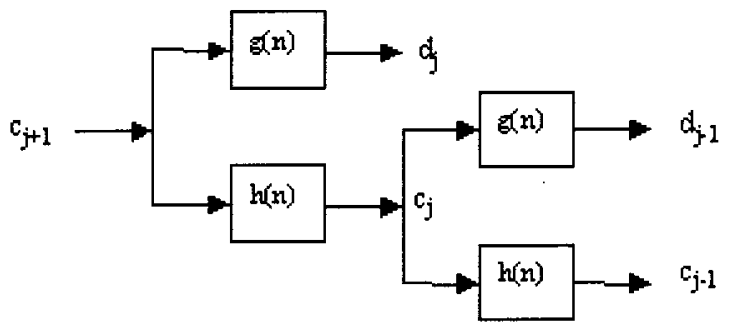

\section{Synthesis}

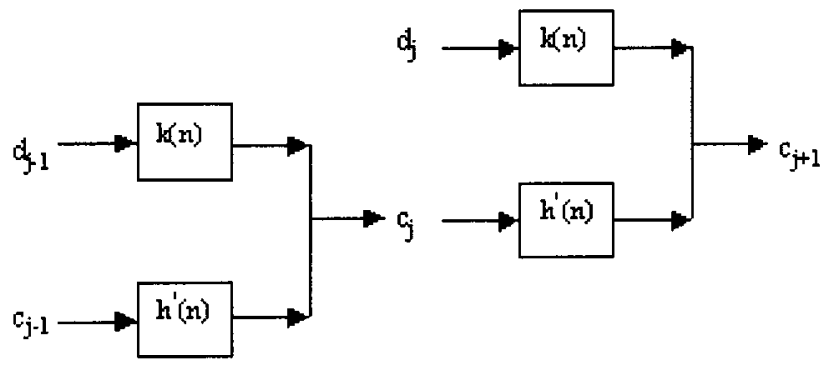

Fig. 9. An over-complete wavelet expansion (upper) and synthesis (lower). Traditional down-sampling during the expansion and up-sampling during the synthesis do not occur.

and discrete filters for the wavelet transform used is shown in Fig. 9.

2) Wavelet Denoising Algorithms: We now describe the multidimensional denoising algorithms and protocols applied in our study. The building block of such denoising algorithms was first introduced by Johnstone and Donoho [16]-[18], [26]. Wavelet coefficients at the finest scale underwent a soft thresholding operation. The "VisuShrink" algorithm determined a threshold $(\lambda)$ according to the sampling number (n) and local statistics of wavelet coefficients (Eqs. (16) and (17), MAD: median absolute value of wavelet modulus). Then, a "soft thresholding" process reduced the amplitudes of the coefficients by $\lambda$. Coefficients whose absolute values were less than $\lambda$ were set to zero. A signal was then reconstructed with the shrunken set of coefficients (18)

$$
\begin{aligned}
\lambda & =\sigma * \operatorname{sqrt}(2 * \log (n)) \\
\sigma^{\wedge} & =\operatorname{MAD} / 0.6745 \\
\eta_{\lambda}(x) & =\left\{\begin{array}{ll}
x-\lambda, & x>=\lambda \\
0, & |x|<\lambda \\
x+\lambda, & x<=-\lambda
\end{array} .\right.
\end{aligned}
$$

In the next section, we describe our multidimensional experimental denoising protocols investigated in terms of quantitative performance.

3) Denoising Protocols: The quantification process using ${ }^{82} \mathrm{Rb}$ images and the two-compartment model is referred as the "original protocol" because no denoising method was applied to images and observed activity matrices. However, as shown in Fig. 8, the flow pattern derived from the original protocol was heterogeneous and contradicted to what should have happened in a heart. This motivated the design of an experiment where wavelet denoising was applied to remove noise in such PET images and dynamic data so as to improve the validity of quantification.

Five denoising protocols were specifically designed to remove noise inherent in ${ }^{82} \mathrm{Rb}$ derived images and observed activity matrices.

"Denoising protocol I" assumed that errors in the dynamic curves mainly resulted from random noise in each short-axis 2-D image. Therefore, 2-D wavelet denoising was applied to each ${ }^{82} \mathrm{Rb}$ image (i.e., 48 frames for each of the 6-10 mid-ventricular planes) independently. The same ROI derived from the original protocol was reused and a new observed activity matrix was then reconstructed.

"Denoising protocol II" assumed that errors in the dynamic curves came from the noise in individual ROIs that fluctuated over time. Therefore, in addition to processing in "denoising protocol I", a 1-D wavelet denoising was applied to the blood and tissue time-activity curves. In this protocol, the temporal array of each ROI [as shown in Fig. 4(b)] was processed independently.

"Denoising protocol III" assumed that errors in the dynamic curves were derived from the noise that disrupted the spatial smoothness and continuity of radioactivity among the ROIs. Therefore, in addition to protocol I, a 2-D multiscale denoising was applied to each submatrix of each observed activity matrix independently [Fig. 4(a)].

"Denoising protocol IV" performed ordered sequential denoising on the original image matrices, the temporal array, followed by processing in the spatial domains of each observed activity matrix. Finally, "denoising protocol V" consisted of ordered sequential denoising on each image, spatial domain processing, followed by temporal domain processing of each observed activity matrix (Table I).

In order to show any advantage of these techniques over conventional methods, classical kernels and frequency filters were empirically selected to smooth PET images and corresponding dynamic curves. The "conventional protocol" was defined as a Butterworth filter with a radius of eight pixels and a reduction factor of $50 \%$ on each $64 \times 64$ PET image and a $3 \times 3$ mean filter in the spatial domain of each observed activity matrix (Table I). This approach was shown superior to other empirical methods in achieving accurate quantification and, therefore, was chosen as a fair comparison to the state-of-the-art wavelet protocols.

Tissue time-activity curves in the reconstructed observed activity matrices derived from the five denoising protocols and the conventional protocol were fitted to the same two-compartment model to obtain flow estimates.

Quantitative results derived from the application of each protocol were evaluated according to their precision, accuracy, and differentiating capacity. In the following section, we describe the evaluation of these experiments.

\section{Performance Evaluation}

1) Precision: Precision of a flow estimate was defined as the ratio of the point estimate over its associated standard error. Since protocols in each ${ }^{82} \mathrm{Rb}$ study quantified flows in $8 \times$ $N$ ROIs $(N:\{6,7,8,9,10\}$, the number of mid-ventricular planes), there were as many estimate-to-error ratios (EERs). The 
TABLE I

Summary of the Original, Five Denoising Protocols, and Conventional Protocol, Applied to Images and ObServed Activity MATRICES (O. C.: OVER-COMPLETE)

\begin{tabular}{|c|c|c|c|c|}
\hline \multirow[t]{3}{*}{ Protocols } & \multirow{3}{*}{$\begin{array}{c}\text { Processing Sequence: } \\
\text { Denoising Strategy }\end{array}$} & \multicolumn{3}{|c|}{ Denoising Kernels } \\
\hline & & Image & \multicolumn{2}{|c|}{ Observed Activity Matrix } \\
\hline & & & Temporal Domain & Spatial Domain \\
\hline Original Protocol & none & none & none & $\overline{\text { none }}$ \\
\hline Wavelet Protocol I & short-axis image & o. c. wavelet & no & $\overline{\text { no }}$ \\
\hline Wavelet Protocol II & image $\rightarrow$ temporal array & o. c. wavelet & o. c. wavelet & $\overline{\text { no }}$ \\
\hline Wavelet Protocol III & image $->$ spatial continuity & o. c. wavelet & no & o. c. wavelet \\
\hline Wavelet Protocol IV & image $->$ temporal $->$ spatial & o. c. wavelet & o. c. wavelet & o. c. wavelet \\
\hline Wavelet Protocol V & image $->$ spatial $\rightarrow>$ temporal & o. c. wavelet & o. c. wavelet & o. c. wavelet \\
\hline Conventional Protocol & image -> spatial & Butterworth & no & mean filter \\
\hline
\end{tabular}

median of the EERs in all ROIs was used as an indicator of precision associated with a specific protocol.

2) Accuracy: Flow estimates derived from each ${ }^{82} \mathrm{Rb}$ protocol were compared with an acknowledged "gold standard," i.e., reference values derived from $\mathrm{H}_{2}^{15} \mathrm{O}$ studies. The means and standard deviations of all regional differences were calculated for comparison.

3) Differentiating Capacity: Quantitative results from patient studies were used to evaluate the differentiating capacity of each protocol. A discriminating protocol must be able to separate regions with normal perfusion from ischemia. Therefore, a functional polar map must visibly show a delineation between normal and infarcted regions. The distribution pattern of cumulative radioactivity (count-based polar map) and absolute flows (functional polar map) were visually compared. Pearson's correlation between counts and flows was measured for each patient. The correlation coefficient was transformed to a $z$ value, as shown in (19). The $z$ values derived from different denoising protocols were statistically compared with the original protocol using a paired $t$-test

$$
z=\log ((1+r) /(1-r))
$$

where $r$ is Pearson's correlation coefficient.

\section{RESULTS}

\section{A. Precision}

The median EERs derived from the original protocol were $2.28 \pm 0.65$ (Fig. 10, $n=17$ ). A typical normal perfusion was near $1.0 \mathrm{ml} / \mathrm{g} / \mathrm{min}$; therefore, its standard error ranged from 0.34 to $0.61 \mathrm{ml} / \mathrm{g} / \mathrm{min}$. The degree of uncertainty is too high to provide valid measurements in clinical practice. Denoising protocol $\mathrm{V}$ resulted in the highest precision $(10.70 \pm 3.45)$ and reached a gain of $4.85(p<0.001)$ compared with the original protocol. With a flow of $1.0 \mathrm{ml} / \mathrm{g} / \mathrm{min}$, its standard error dropped to $0.07-0.14 \mathrm{ml} / \mathrm{g} / \mathrm{min}$, small enough to differentiate between normal perfusion and ischemia in clinical practice.

\section{B. Accuracy}

Regional differences between the flow estimates derived from each ${ }^{82} \mathrm{Rb}$ protocol and the "gold standard" obtained from $\mathrm{H}_{2}^{15} \mathrm{O}$ studies are shown in Table II. Although on average, quantification with the original protocol only slightly overestimated myocardial perfusion $(0.05 \pm 0.80 \mathrm{ml} / \mathrm{g} / \mathrm{min})$, its large variation hampered accurate estimation in individual regions. The conventional protocol significantly reduced the variation between the two approaches, but also resulted in underestimates, on average. Denoising protocols III, IV, and V, all of which involved a spatial smoothing and continuity constraint on the observed activity matrix, reduced the standard deviation to the greatest extent with only a slight mean bias (Table II). Thus, these three denoising protocols could be used to measure regional flows accurately.

\section{Differentiating Capacity}

Fig. 7 shows a count-based polar map of a ${ }^{82} \mathrm{Rb}$ scan of a patient [Fig. 7(a)] produced by the Siemens ECAT system at Columbia Presbyterian Medical Center. The color code shows that radioactivity is low in the apex, the anterioseptal, septal, and lateral walls of the heart, i.e., relative hypoperfusion exists in these regions. Fig. 7(b) represents the same count-based polar map divided by sectors defined in the ROI operation. The functional polar map that presents the absolute flow estimates derived from the original protocol and the five denoising protocols are shown in Fig. 11. The conventional protocol was not evaluated in these patients because it involved a mean filter smoothing on the observed activity matrix and inevitably mixed normal and ischemic regions. The functional polar maps derived from the original protocol, denoising protocols I and II (upper left, upper right, and middle left panels of Fig. 11) show significantly varying perfusion, even among adjacent ROIs. Although flows seem higher in the lateral wall, no obvious flow pattern can be identified.

On the contrary, flow estimates with denoising protocols III, $\mathrm{IV}$, and $\mathrm{V}$ (middle right, lower left, and lower right panels of Fig. 11) demonstrate a similar distribution pattern to the countbased polar map shown in Fig. 7. The functional polar maps demonstrate that flow was low in the anterior, septal, and proximal and distal parts of the posterior regions. Absolute values were higher in the lateral wall and some portions of the posteriolateral wall. There was also a high visual correlation between counts and flows; even a small hot spot in the middle level of 


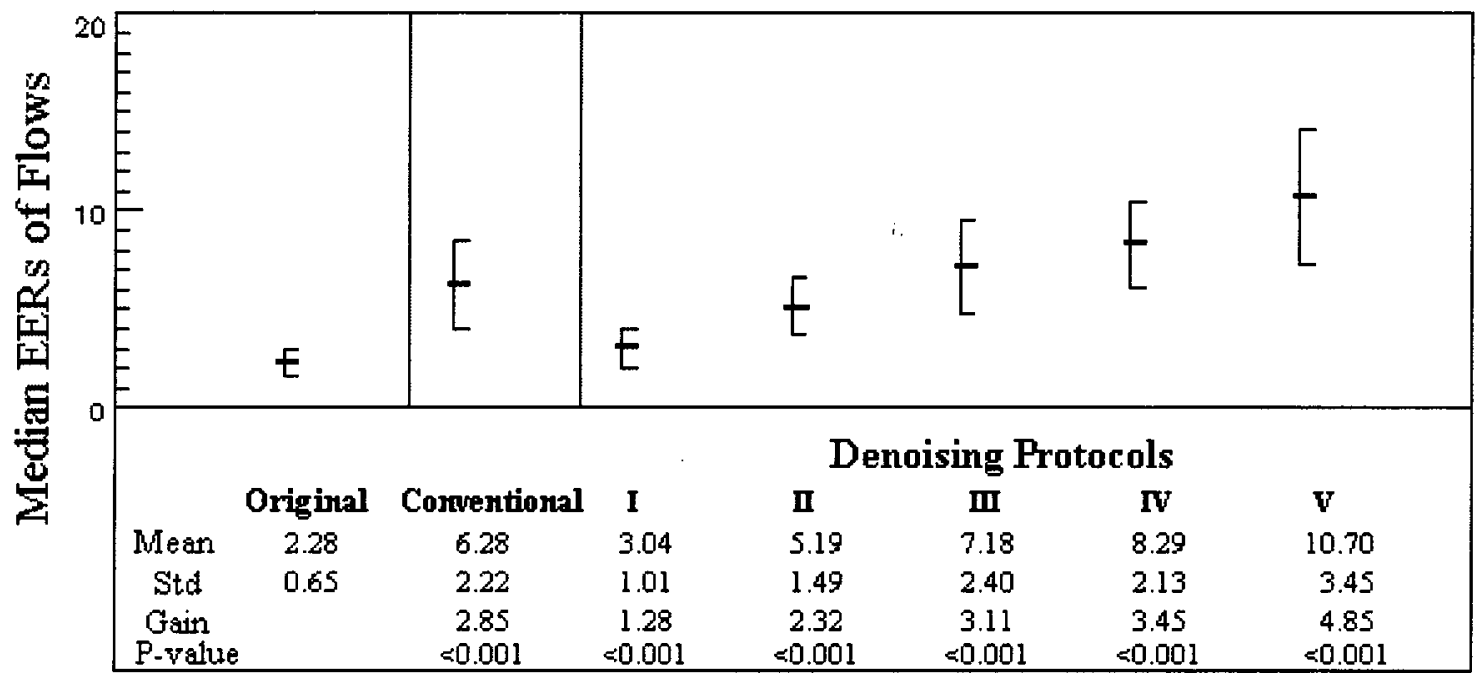

Fig. 10. Box plots (mean \pm standard deviation) of the median EERs of flows with the original protocol ("original"), a conventional protocol ("conventional"), and the five denoising protocols in $17^{82} \mathrm{Rb}$ studies. The conventional method improved precision but not as significantly as denoising protocols III, IV, and V. Denoising protocol $\mathrm{V}$ improved the precision to the greatest extent and achieved a gain of 4.85 compared to the original protocol $(p<0.001)$.

TABLE II

DIFFERENCES IN REGIONAL FLOWS DERIVED FROM DISTINCT ${ }^{82} \mathrm{Rb}$ PROTOCOLS AND THE "GOLD STANDARD” OBTAINED FROM His $\mathrm{H}_{2}^{15}$ O STUDIES.

DENOISING PROTOCOLS III, IV, AND V SIGNIFICANTLY REDUCED THE STANDARD DEVIATION OF THE DifFERENCES WITH A SMALl BIAS

\begin{tabular}{l|r}
\hline \multicolumn{1}{c|}{ Protocols } & \multicolumn{1}{c}{$\begin{array}{c}\text { Differences } \\
\text { compared to Gold } \\
\text { Standard } \\
(\mathrm{ml} / \mathrm{g} / \mathrm{min}, \\
\mathrm{n}=560 \mathrm{ROIs})\end{array}$} \\
\hline Original Protocol & $0.05 \pm \mathbf{0 . 8 0}$ \\
\hline Conventional Protocol & $-0.1 \mathbf{0 . 0 . 2 2}$ \\
\hline Denoising Protocol I & $-0.06 \pm \mathbf{0 . 6 0}$ \\
Denoising Protocol II & $0.01 \pm \mathbf{0 . 5 0}$ \\
Denoising Protocol III & $0.06 \pm \mathbf{0 . 2 0}$ \\
Denoising Protocol IV & $-0.03 \pm \mathbf{0 . 2 0}$ \\
Denoising Protocol V & $-0.09 \pm \mathbf{0 . 2 0}$ \\
\hline
\end{tabular}

the lateral wall was associated with high flow. Therefore, each of these three denoising protocols maintained and discerned the flow pattern in diseased hearts.

The similarity between counts and flows was quantified by the $z$ transformation of Pearson's correlation coefficients (19). With the original protocol, the $z$ values of the 13 patients were $0.58 \pm 0.60$. With denoising protocols III, IV, and V, the $z$ values were significantly elevated $(p<0.001)$ (Fig. 12). These three protocols which involved denoising in the spatial domain of the observed activity matrix improved the correlation between counts and flows. Thus, protocols III, IV and V also improved the differentiation between normal and hypo-perfused regions.

\section{DISCUSSION}

Although PET is a powerful tool for analyzing a variety of physiological functions, this study demonstrated that quantification of myocardial perfusion using uncorrected dynamic data cannot produce accurate estimates when dynamic curves are noisy. A multiscale method of analysis recovered multidimensional signals deeply hidden within spatial/temporal "stacks" of PET-acquired images. This study used dynamic PET images with ${ }^{82} \mathrm{Rb}$ as an example to evaluate the effects of wavelet-based signal detection/denoising for the use of physiological quantification and then verified the capacity of this method to achieve precision, accuracy, and differentiation in determining myocardial perfusion. A wavelet transformation has the ability to adapt to local variation and also provides a theoretical framework for finding a near-optimal estimate to an underlying function. We showed that this approach can spot and preserve rapid changes in cardiac signals and separate affected and nonaffected regions.

The denoising protocols developed and applied in this study assumed uncorrelated errors among pixels, in the temporal and spatial domains of the observed activity matrix. However, the denoising performance remained satisfactory and robust even if correlated errors existed. There are several ways to explain this phenomenon. First, the original error-prone signals were decomposed into multiple planes, including the $x$ and $y$ axes of the original image matrix, the horizontal and vertical axes along regions, and the temporal direction. When errors are decomposed onto orthogonal planes, it is possible to manage them as uncorrelated measures in each dimension. Second, in each short-axis image, clusters of positive and negative residuals were frequently observed. Therefore, the errors among adjacent pixels were correlated according to their geometric positions. However, when the pixels were grouped into ROIs, the residuals, viewed from either the temporal or the spatial dimension, became normally distributed. The occurrence of clustered residuals among pixels could be regarded as random fluctuations when radioactivity was considered from a multidimensional view on a region-by-region basis. Third, due to the central limit theory, the averaging effects of pixel activities from any unknown distribution become normal when repeated sampling is performed. Therefore, it is likely that the residuals in the spatial and the temporal dimensions of the residual activity matrix were indeed uncorrelated and normal. 


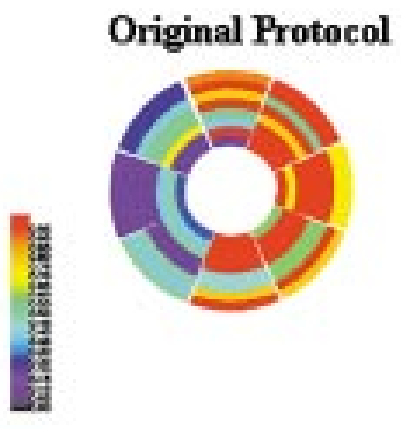

Denoising Protocol II

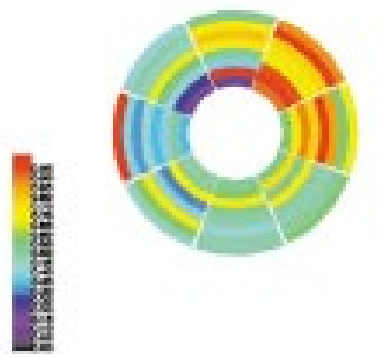

Denoising Protocol IV

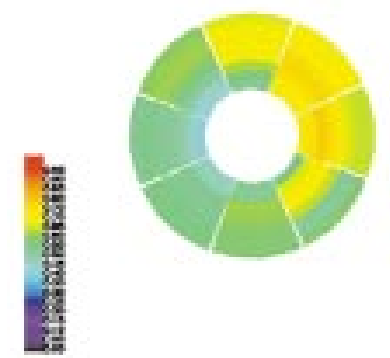

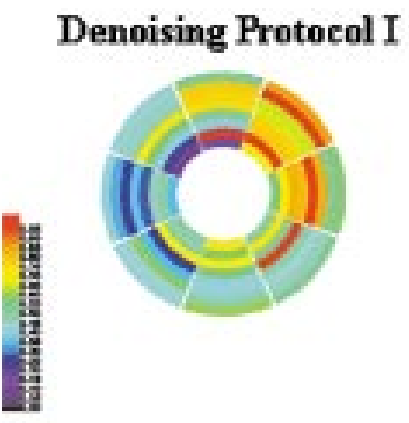

Denoising Protocol III

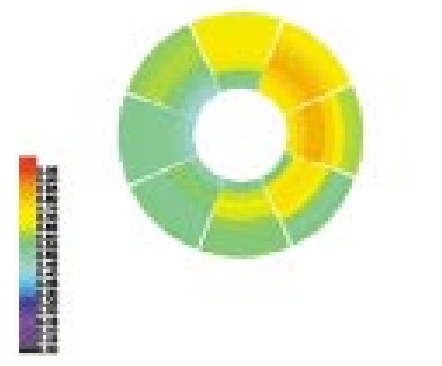

Denoising Protocol V

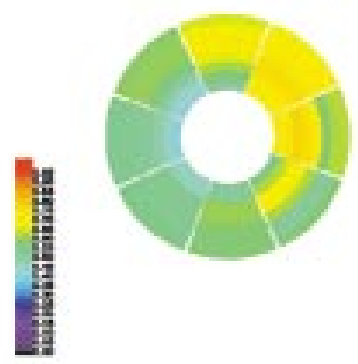

Fig. 11. The functional polar maps of a sample patient derived from the original protocol and the five denoising protocols. The flow distribution obtained from denoising protocols III, IV, and V was similar to that of the count-based polar map shown in Fig. 7.

The varying performances of the five denoising protocols suggest that the true errors in observed activity matrices include a major component from the spatial domain and a minor contribution from the temporal domain. Simply removing random noise in the short-axis images (denoising protocol I) did not improve the validity of the quantification process. Indeed, independent smoothing on the time-activity curve of each ROI neither complied with the true error nor incorporated geometric information and, therefore, resulted in the poor performance of denoising protocol II and introduced bias in the first step of denoising protocol IV. In addition, a pseudothree-dimensional (1-D temporal +2 -D spatial) noise reduction, denoising protocol IV, produced only slightly more precise estimates than denoising protocol III. On the other hand, denoising protocol III, with only spatial noise reduction, performed as well as denoising protocols IV and V in accuracy and diagnostic capacity. Thus, we claim that the first step in denoising protocol $\mathrm{V}$ removed most of the errors, and the second step further reduced the distance between the tissue curve and the true underlying signal, yielding more precise estimates of local perfusion.
Errors that perturbed spatial continuity of radioactivity among ROIs originated from many sources. In a temporal frame of $5 \mathrm{~s}$, there may be four heartbeats and one respiration cycle. Thus, the radioactivity of each pixel reflects an average of a moving object. In addition, the template of ROIs was also a major source of error. In the ROI analysis, the same template that fixed the coordinates of each selected region was applied to all frames. In a typical ${ }^{82} \mathrm{Rb}$ study, direct visualization of the heart in each frame was impossible due to the ever-changing tracer kinetics and low SNRs. During the scan period, the heart expanded, contracted, rotated, and moved in oblique directions. The application of the same template to each frame results in a disruption of continuity among regions. Denoising protocols III, IV, and V all involved a spatial smoothing of the observed activity matrix and effectively compensated for violation of continuity. Thus, these three protocols significantly improved the precision, accuracy, and differentiating capacity of the quantification process. Denoising protocol V out-performed the other two only in terms of precision with a gain of 1.50 . 


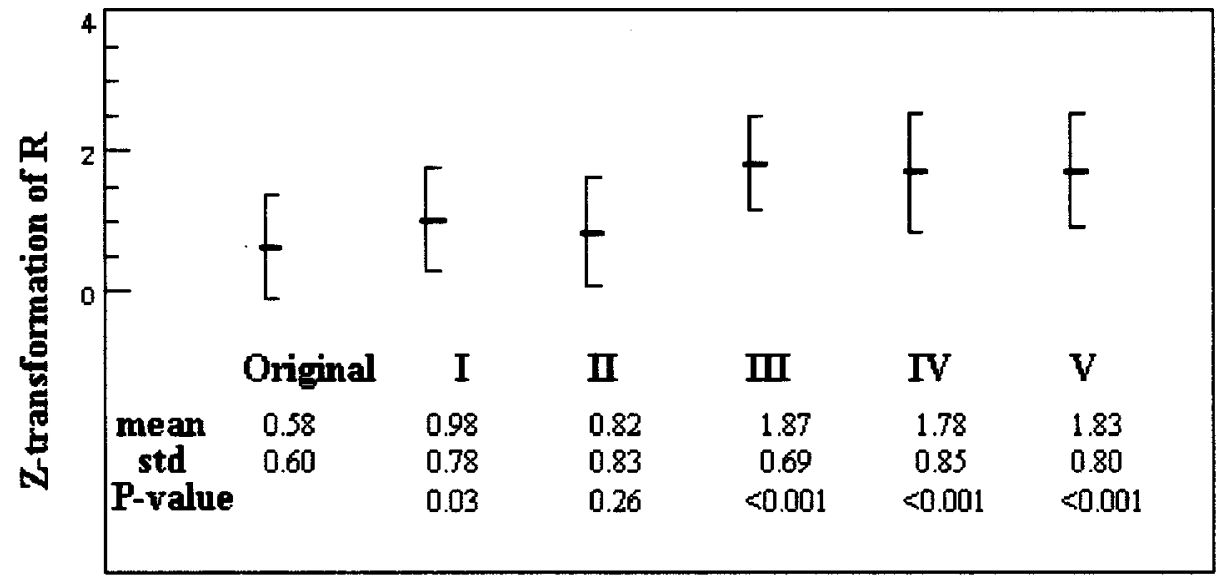

Fig. 12. Box plots (mean \pm standard deviation) on the $z$ transformation of correlation coefficients between cumulative counts and absolute flow estimates derived from the original protocol and the five denoising protocols applied to each patient. The conventional protocol was not evaluated in these patients because it involved mean filtering on the observed activity matrix and mixed normal and ischemic regions. Denoising protocols III, IV, and V significantly increased the correlation between counts and flows $(p<0.001)$ and, therefore, was able to describe the flow distribution in diseased hearts.

This study, for the first time, introduces principles of wavelet theory and wavelet-based denoising methods into image-based physiological quantification. Multiscale analysis provided a robust method for revealing functional information, in a vast amount of spatial/temporal data. Previously, quantification of physiological function could not provide reliable functional data because estimates of target regions appeared solitary and unrelated to each other. Only after the application of these denoising protocols, which removed uncorrelated noise both in the spatial and temporal dimensions of contiguous ROIs, could image-based quantification produce accurate and precise estimates of perfusion.

\section{CONCLUSION}

This study evaluated the use of multiscale wavelet transforms on image-based physiological quantification, and provides important information for clinical application. We demonstrated that noninvasive quantification by using dynamic PET images with ${ }^{82} \mathrm{Rb}$ accurately measured myocardial perfusion in human subjects. In clinical applications, this approach should be able to be generalized to other dynamic image modalities with similar tracers or contrast media to quantify various physiological or pathological processes. Continued research in the fields of mathematics and biomedical engineering may lead to new developments in wavelet theory and applications including alternative bases of expansion. Clinical use of these methods can improve the estimation of functional parameters in a wide array of spatial/temporal imaging modalities in medicine.

\section{REFERENCES}

[1] M. E. Phelps, J. C. Mazziotta, and H. R. Schelbert, Positron Emission Tomography and Autoradiography-Principles and Applications for the Brain and Heart. New York: Raven, 1986.

[2] K. Godfrey, Compartmental Models and Their Application, London, U.K.: AcademicLTD, 1983.

[3] C. R. Bellina, O. Parodi, P. Camici, P. A. Salvadori, L. Taddei, L. Fusani, R. Guzzardi, G. A. Klassen, A. L. L'Abbate, and L. Donato, "Simultaneous in vitro and in vivo validation of nitrogen-13-ammonia for the assessment of regional myocardial blood flow," J. Nucl. Med., vol. 31, pp. $1335-1343,1990$
[4] G. D. Hutchins, M. Schwaiger, K. C. Rosenspire, J. Krivokapich, H. Schelbert, and D. E. Kuhl, "Noninvasive quantification of regional blood flow in the human heart using $\mathrm{N}-13$ ammonia and dynamic positron emission tomographic imaging," J. Amer. Coll. Cardiol., vol. 15, pp. 1032-1042, 1990.

[5] P. Herrero, J. Markham, M. E. Shelton, C. J. Weinheimer, and S. R. Bergmann, "Noninvasive quantification of regional myocardial perfusion with rubidium-82 and positron emission tomography. Exploration of a mathematical model," Circulation, vol. 82, pp. 1377-1386, 1990.

[6] P. Herrero, J. Markham, M. E. Shelton, and S. R. Bergmann, "Implementation and evaluation of a two-compartment model for quantification of myocardial perfusion with rubidium-82 and positron emission tomography," Circ. Res., vol. 70, pp. 496-507, 1992.

[7] W. G. Kuhle, G. Porenta, S. C. Huang, D. Buxton, S. S. Gambhir, H. Hansen, M. E. Phelps, and H. R. Schelbert, "Quantification of regional myocardial blood flow using ${ }^{13} \mathrm{~N}$-ammonia and reoriented dynamic positron emission tomographic imaging," Circulation, vol. 86, pp. 1004-1017, 1992.

[8] S. R. Bergmann, K. A. Fox, A. L. Rand, K. D. McElvany, M. J. Welch, J. Markham, and B. E. Sobel, "Quantification of regional myocardial blood flow in vivo with $\mathrm{H}_{2}^{15} \mathrm{O}$," Circulation, vol. 70, pp. 724-733, 1984.

[9] P. Herrero, J. Markham, and S. R. Bergmann, "Quantitation of myocardial blood flow with $\mathrm{H}_{2}^{15} \mathrm{O}$ and positron emission tomography: Assessment and error analysis of a mathematical approach," J. Comput. Assist. Tomogr, vol. 13, pp. 862-873, 1989.

[10] P. Horlock, J. Clark, H. O'Brien, P. Grant, and A. Bentley, “The preparation of a rubidium-82 radionuclide generator," J. Radioanal. Chem., vol. 64, pp. 257-265, 1981.

[11] S. C. Huang, B. A. Williams, J. Krivokapich, L. Araujo, M. E. Phelps, and H. R. Schelbert, "Rabbit myocardial ${ }^{82} \mathrm{Rb}$ kinetics and a compartmental model for blood flow estimation," Amer. J. Physiol., vol. 256, pp. H1156-H1164, 1989.

[12] M. Grover-McKay, O. Ratib, M. Schwaiger, D. Wohlgelernter, L. Araujo, C. Nienaber, M. Phelps, and H. R. Schelbert, "Detection of coronary artery disease with positron emission tomography and rubidium-82," Amer. Heart. J., vol. 123, pp. 646-652, 1992.

[13] A. F. Laine, S. Schuler, J. Fan, and W. Huda, "Mammographic feature enhancement by multiscale analysis," IEEE Trans. Med. Imag., vol. 13, pp. 725-740, Dec. 1994.

[14] A. F. Laine, J. Fan, and W. Yang, "Wavelets for contrast enhancement of digital mammography," IEEE Eng. Medicine Biol. Soc. Mag. (Wavelets for Image Analysis), vol. 14, pp. 536-550, Sept./Oct. 1995.

[15] K. Herholz, "Non-stationary spatial filtering and accelerated curve fitting for parametric imaging with dynamic PET," Eur. J. Nucl. Med., vol. 14, pp. 477-484, 1988.

[16] D. L. Donoho and I. M. Johnstone, "Adapting to unknown smoothness via wavelet shrinkage," J. Amer. Stat. Assoc., vol. 90, pp. 1200-1223, 1995.

[17] D. L. Donoho, "De-noising by soft-thresholding," IEEE Trans. Inform. Theory, vol. 41, pp. 613-627, May 1995. 
[18] D. L. Donoho and I. M. Johnstone, "Ideal spatial adaptation by wavelet shrinkage," Biometrika, vol. 81, pp. 425-455, 1994

[19] Y. Choi, S. C. Huang, R. A. Hawkins, W. G. Kuhle, M. Dahlbom, C. K. Hoh, J. Czernin, M. E. Phelps, and H. R. Schelbert, "A simplified method for quantification of myocardial blood flow using nitrogen-13ammonia and dynamic PET," J. Nucl. Med., vol. 34, pp. 488-497, 1993.

[20] R. H. Huesman and B. M. Mazoyer, "Kinetic data analysis with a noisy input function," Phys. Med. Biol., vol. 32, pp. 1569-1579, 1987.

[21] D. W. Marquardt, "An algorithm for least-squares estimation of nonlinear parameters," J. Soc. Ind.. Appl. Math., vol. 11, pp. 431-441, 1963.

[22] I. Koren and A. Laine, "A discrete dyadic wavelet transform for multidimensional feature analysis," in Time-Frequency and Wavelet Transforms in Biomedical Engineering. Piscataway, NJ: IEEE Press, 1997.

[23] X. Zong, A. F. Laine, and E. A. Geiser, "Speckle reduction and contrast enhancement of echocardiograms via multiscale nonlinear processing," IEEE Trans. Med. Imag., vol. 17, pp. 532-540, Aug. 1998.

[24] C. S. Burrus, R. A. Gopinath, and H. Gauo, Introduction to Wavelets and Wavelet Transforms. Englewood Cliffs, NJ: Prentice-Hall, 1998.

[25] S. Mallat and S. Zhong, "Characterization of signals from multiscale edges," IEEE Trans. Pattern Anal. Machine Intell., vol. 14, pp. 710-732, July 1992.

[26] D. L. Donoho and I. M. Johnstone, "Threshold selection for wavelet shrinkage of noisy data," in Proc. 1994 IEEE Conf. Image Processing, vol. 1, 1994, pp. A24-A25.

[27] — - "Ideal spatial adaptation by wavelet shrinkage," Biometrika, vol. 81 , no. 3, pp. 425-455, 1994a.

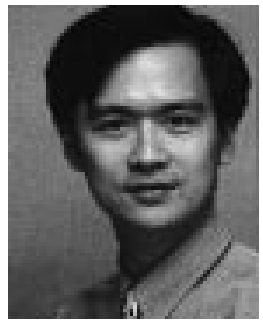

Jou-Wei Lin was born in March 3, 1968. He received the M.D. degree from National Taiwan University Hospital, the M.P.H. degree from Harvard University, Cambridge, MA, and the Ph.D. degree from the Department of Medical Informatics, Columbia University, New York, NY.

$\mathrm{He}$ is currently an Internist at National Taiwan University Hospital, Taipei, Taiwan. He is interested in computer applications in medical care, especially in data mining of clinical information from various forms of medical data, including images and

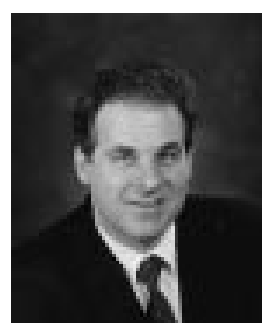

Andrew F. Laine received D.Sc. degree in computer science, from the School of Engineering and Applied Science, Washington University, St. Louis, MO, in 1989 and B.S. degree from Cornell University, Ithaca, NY, in 1977.

He joined the Department of Computer and Information Sciences and Engineering at the University of Florida, Gainesville, in 1990 as an Assistant Professor. In 1997 he moved to Columbia University, New York, NY, as an Associate Professor of Biomedical Engineering and Radiology, and Director of the Biomedical Imaging Laboratory. He pioneered the application of multiresolution representations for feature analysis of digital mammography and ultrasound. He served Associate Editor of IEEE TRANSACTIONS ON IMAGE PrOCESSING and edited the book, Wavelet Theory and Applications (Norwell, MA: Kluwer, 1995). He has chaired the conference on "Mathematical Imaging: Wavelet Application in Signal and Image Processing," during the years 1993-2000. He co-authored chapters in the books Wavelet Applications in Medicine and Biology (Boca Raton, FL: CRC Press, 1995), and Time-Frequency and Wavelets Transforms in Biomedical Engineering (Piscataway, NJ: IEEE Press, 1998). His research interests include methods of multiresolution analysis applied to problems in medical imaging, image processing, computed aided diagnosis, pattern recognition, and applied mathematics.

Dr. Laine is a member of the Editorial Board of the book series Emerging Technologies in Biomedical Engineering, sponsored by the IEEE-EMBS Society. He also served on the program committee for the IEEE-EMBS Workshop on Wavelet Applications in Medicine in 1994, 1998, and 1999. He is an active member of the IEEE Computer Society and Engineering in Medicine and Biology Society.

Steven R. Bergmann received the B.A. degree in 1972 from the George Washington University, Washington, D.C., and the Ph.D. degree in physiology and biophysics in 1978 from the Hahnemann Medical College, Philadelphia, PA. He received the M.D. degree from Washington University School of Medicine in 1985.

He was a Faculty Member in the Cardiovascular Division, Department of Internal Medicine, from 1979-1996. In 1996, he joined the Department of Medicine, Division of Cardiology at the College of Physicians and Surgeons of Columbia University, New York, NY, where he is currently Professor of Medicine and Radiology and Director of Nuclear Cardiology. His research interests include the use of positron emission tomography for the quantification of myocardial flow and metabolism in the assessment of cardiac disease, and evaluation of the efficacy of therapeutic interventions designed to mitigate myocardial dysfunction. 